\title{
Entropy Boundary and Jump Conditions in the Theory of Sedimentation with Compression
}

\author{
R. Bürger and W. L. Wendland*
}

Institute of Mathematics A, University of Stuttgart, Pfaffenwaldring 57, D-70569 Stuttgart, Germany

Dedicated to Willi Törnig on the occasion of his retirement

\begin{abstract}
We present an initial-boundary value problem of a quasilinear degenerate parabolic equation for the settling and consolidation of a flocculated suspension. The corresponding definition of generalized solutions is formulated. It is based on an entropy integral inequality in the sense of Kružkov. From this definition, jump and entropy conditions that have to be satisfied at discontinuities, and an entropy condition valid on one boundary of the computational domain are derived. The latter implies a set-valued reformulation of the original boundary condition. It is interpreted geometrically and characterized by the solution of an auxiliary hyperbolic Riemann problem. (C) 1998 B. G. Teubner Stuttgart-John Wiley \& Sons, Ltd.
\end{abstract}

\section{Introduction}

We analyse a mathematical model of industrial sedimentation processes in which a flocculated suspension is separated into its solid and liquid parts under the influence of gravity. The model is based on a general phenomenological theory of flocculated suspensions formulated in the recent years by Concha et al. in References 14 and 15, where only the macroscopical behaviour of the suspension as a mixture of two superimposed continuous media is studied, rather than the settling of individual particles. This theory leads to an initial-boundary value problem of a scalar quasilinear partial differential equation of second-order parabolic type for the local volumetric solid concentration as a function of height and time. The differential equation degenerates into a first-order hyperbolic equation if the concentration is less than a critical value, i.e. on an interval of solution values. Moreover, our mathematical model includes a purely hyperbolic equation corresponding to the classical theory of sedimentation by Kynch [18] and its extensions to continuous sedimentation processes, which are studied thoroughly in References 6, 7, 9, 12 and 13.

The initial-boundary value problem may possess discontinuous solutions, hence an appropriate definition of generalized solutions has to be introduced. Here we follow

*Correspondence to: W. L. Wendland, Institute of Mathematics A, University of Stuttgart, Pfaffenwaldring 57, D-70569 Stuttgart, Germany. 
the work of Vol'pert et al. [23-25] and Zhuoqun Wu et al. [26-29], transferring their results to inhomogeneous boundary conditions of different types at the spatial boundaries of the computational domain. The definition of generalized solutions given here in section 3 is based on Kružkov's definition of Cauchy problems for hyperbolic conservation laws [17] and includes an entropy condition as a selection principle for the physically relevant one among the family of weak solutions.

In this paper, we present a jump condition, valid in the interior of the computational domain, and an entropy boundary condition establishing the relationship between the given boundary data at the spatial domain to the values actually assumed by the generalized solution, which need not coincide. Existence, stability and uniqueness of generalized solutions are shown in Reference 4.

\section{Formulation of the initial-boundary value problem}

\subsection{Assumptions}

The phenomenological theory of flocculated suspensions (see References 3, 14 and 15 for details) emerges from the basic assumptions that the solid particles are small with respect to the sedimentation vessel and have the same density, that the components of the suspension are incompressible, that the suspension is entirely flocculated at the beginning of the sedimentation process and that there is no mass transfer between solid and liquid during sedimentation. Its reduction to one space dimension corresponds to the concept of an ideal continuous thickener (ICT, Fig. 1) as a kinematical constitutive assumption: an ICT is a cylindrical vessel without wall effects in which all field variables are assumed to be constant across each cross-section, so that they

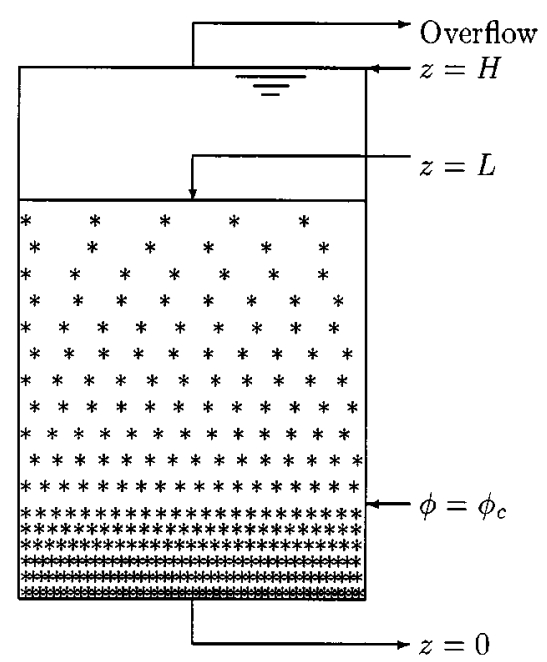

Fig. 1. The ideal continuous thickener (ICT) 
depend only on $z$ and $t$. At height $z=L$, there is a surface source for continuous feeding of the thickener with fresh suspension, and at $z=0$, the sediment is discharged continuously through a surface sink. An ICT without feeding and discharge can be operated as a settling column for batch sedimentation. At the bottom, the sediment forms, and it is assumed that the solid flocs begin to touch each other at a critical concentration value $\phi_{\mathrm{c}}$.

The solid and fluid material behaviour is reflected by the dynamical constitutive assumptions that both solid and fluid components of the mixture are elastic fluids, and that gravitation is the only body force.

\subsection{Field equation}

The basic mass and linear momentum balance laws for the solid and liquid component reduce by the constitutive assumptions, a subsequent dimensional analysis and the introduction of experimental rather than theoretical variables $[3,14]$ to the following continuity equation:

$$
\frac{\partial}{\partial t} \phi(z, t)+\frac{\partial}{\partial z}(\hat{f}(\phi, t))=\frac{\partial}{\partial z}\left(-\hat{f}_{\mathrm{b} k}(\phi) \cdot \frac{\sigma_{\mathrm{e}}^{\prime}(\phi)}{\Delta \varrho \phi g} \cdot \frac{\partial \phi}{\partial z}\right) .
$$

Here $\phi=\phi(z, t)$ is the volumetric solid concentration, $t$ the time, $0 \leqslant z \leqslant L$ the height, $\hat{f}_{\mathrm{b} k}(\phi)$ the solid flux density function corresponding to batch sedimentation processes, $\sigma_{\mathrm{e}}(\phi)$ the effective solid stress, $\Delta \varrho$ the difference of solid and fluid mass densities, $g$ the acceleration of gravity, $\hat{q}(t)$ the average flow velocity of the suspension which can be controlled externally, and

$$
\hat{f}(\phi, t)=\hat{q}(t) \phi+\hat{f}_{\mathrm{b} k}(\phi) .
$$

We assume that $\hat{f}_{\mathrm{b} k}$ is a twice continuously differentiable function satisfying

$$
\hat{f}_{\mathrm{b} k}(0)=\hat{f}_{\mathrm{b} k}(1)=0, \quad \hat{f}_{\mathrm{b} k} \leqslant 0 \text { for } 0 \leqslant \phi \leqslant 1, \hat{f}_{\mathrm{b} k}^{\prime}(1)=0,
$$

where we put formally

$$
\hat{f}_{\mathrm{b} k}(\phi)=0 \text { for } \phi<0 \text { or } \phi>1
$$

and assume that $\hat{q}$ satisfies

$$
\hat{q} \in C^{1}[0, T], \quad \hat{q}(t) \leqslant 0 .
$$

In practice, the formula by Richardson and Zaki [21] is employed frequently, which leads to

$$
\hat{f}_{\mathrm{b} k}(\phi)=v_{\infty} \cdot \phi \cdot(1-\phi)^{C+1} \quad \text { with } C>0,
$$

where $v_{\infty}$ is the flow velocity of a singular particle in an unbounded medium. Hindered settling takes place where the solid particles do not touch each other, i.e. $\phi(z, t)<\phi_{\mathrm{c}}$, and consolidation or compression regions correspond to $\phi(z, t) \geqslant \phi_{\mathrm{c}}$. We assume that in hindered settling regions no solid stress exists, i.e. $\sigma_{\mathrm{e}}=0$ for $\phi<\phi_{\mathrm{c}}$, and that $\sigma_{\mathrm{e}}(\phi)$ is a nonnegative nondecreasing twice continuously differentiable function for $\phi \geqslant \phi_{\mathrm{c}}$. Thus, equation (2.1) is a quasilinear parabolic partial differential equation which is 
degenerate in the sense that it is of hyperbolic type for $\phi \leqslant \phi_{\mathrm{c}}$ and $\phi \geqslant 1$; and of parabolic type for $\phi_{\mathrm{c}}<\phi<1$. In general, the location of the interface $\phi(z, t)=\phi_{\mathrm{c}}$ is not known beforehand. For $\phi<\phi_{\mathrm{c}}$, we obtain the hyperbolic conservation law of the extension of Kynch's sedimentation model [18] to continuous sedimentation processes $[6,7,9,10,12,13]$. In References 5 and 15, the following discontinuous constitutive law for $\sigma_{\mathrm{e}}(\phi)$ is suggested:

$$
\sigma_{\mathrm{e}}(\phi)=\left\{\begin{array}{cl}
0 & \text { for } \phi<\phi_{\mathrm{c}}, \\
a \mathrm{e}^{b \phi} & \text { for } \phi \geqslant \phi_{\mathrm{c}} .
\end{array}\right.
$$

Here, $a$ and $b$ are parameters to be obtained from measurements. However, several experimental results $[2,22]$ justify a smooth law for $\sigma_{\mathrm{e}}(\phi)$ by physical arguments. For a discontinuous effective solid stress function $\sigma_{\mathrm{e}}$, certain results of the analysis of the mathematical model derived here can be shown to remain valid (cf e.g. Reference 11), but for the existence proof, twice differentiability of $\sigma_{\mathrm{e}}(\phi)$ has been indispensable so far. Furthermore, the assumptions on $\sigma_{\mathrm{e}}$ are chosen in such a way that the purely hyperbolic case, $\sigma_{\mathrm{e}} \equiv 0$, is included, see Reference 25 . In Reference 8, a flocculated suspension of water and tailings from copper mining was analysed, and the parameters $v_{\infty}=-6.05 \times 10^{-4} \mathrm{~m} / \mathrm{s}$ and $C=11.59$ were obtained for the model (2.6), and the parameters $g=9.8 \mathrm{~m} / \mathrm{s}^{2}, L=6.0 \mathrm{~m}, \varrho_{\mathrm{f}}=1000 \mathrm{~kg} / \mathrm{m}^{3}$ and $\varrho_{s}=2500 \mathrm{~kg} / \mathrm{m}^{3}$ were chosen or measured. For $\sigma_{\mathrm{e}}$, the relation (2.7) was selected, comparison with experimental measurements yielded $\phi_{\mathrm{c}}=0.23, a=5.35 \mathrm{~N} / \mathrm{m}^{2}$ and $b=1.79$.

\subsection{Initial and boundary conditions}

We assume that at the time $t=0$ an initial concentration distribution

$$
\phi(z, 0)=\phi_{0}(z), \quad z \in[0, L]
$$

satisfying

$$
0 \leqslant \phi_{0} \leqslant 1, \quad \phi \in C^{1}[0, \delta) \cap C^{2}(L-\delta, L], \quad \delta>0
$$

is given. In the neighbourhood of $z=0$ and $L$, we require differentiability in order to meet the compatibility conditions of initial and boundary data, which are formulated below. We assume that the control of the feeding flux is equivalent to prescribing explicit concentration values in the thickener at feeding height $z=L$ for $t \in[0, T]$, hence at $z=L$ we require that

$$
\phi(L, t)=\phi_{L}(t), \quad t \in[0, T],
$$

where

$$
\phi_{L} \in C^{1}[0, T], \quad 0 \leqslant \phi_{L}(t) \leqslant 1 .
$$

However, due to the entropy boundary conditions, it will turn out that prescribed concentration values at $z=L$ are not always assumed by the generalized solution, which occurs if the thickener overflows (see Reference 7 for continuous sedimentation without compression). Next, we assume that at $z=0$ the solid volume flux per 
thickener unit area reduces to the fraction that corresponds to the control of the average flow velocity of the suspension by regulating the discharge, i.e.

$$
\hat{f}(\phi, t)+\left.\hat{f}_{\mathrm{b} k}(\phi) \cdot \frac{\sigma_{\mathrm{e}}^{\prime}(\phi)}{\Delta \varrho \phi g} \cdot \frac{\partial \phi}{\partial z}\right|_{z=0}=\left.\hat{q}(t) \phi\right|_{z=0}
$$

which is equivalent to

$$
\left.\hat{f}_{\mathrm{b} k}(\phi)\left(1+\frac{\sigma_{\mathrm{e}}^{\prime}(\phi)}{\Delta \varrho \cdot \phi \cdot g} \cdot \frac{\partial \phi}{\partial z}\right)\right|_{z=0}=0 .
$$

\subsection{Rescaling of the spatial variable}

Introducing $x:=z / L, \quad u(x, t):=\phi(L x, t), \quad q(t):=\hat{q}(t) / L, \quad \bar{u}_{1}(t):=\phi_{1}(t), \quad u_{0}(x):=$ $\phi_{0}(L x), \hat{f}_{\mathrm{b} k}(u):=\hat{f}_{\mathrm{b} k}(u) / L, f(u, t):=q(t) u+\hat{f}_{\mathrm{b} k}(u)$ and

$$
a(u):=-f_{\mathrm{b} k}(u) \frac{\sigma_{\mathrm{e}}^{\prime}(u)}{L \cdot \Delta \varrho \cdot g \cdot u}, \quad \text { i.e. } a(u) \begin{cases}=0, & u \leqslant \phi_{\mathrm{c}}, \\ >0, & \phi_{\mathrm{c}}<u<1, \\ =0, & u \geqslant 1,\end{cases}
$$

the initial-boundary value problem (2.1), (2.8), (2.10), (2.13) for $u=u(x, t)$ can be rewritten as

$$
\begin{aligned}
& \frac{\partial u}{\partial t}+\frac{\partial}{\partial x} f(u, t)=\frac{\partial}{\partial x}\left(a(u) \frac{\partial u}{\partial x}\right) \quad \text { on } Q_{T}=(0,1) \times(0, T), \\
& u(x, 0)=u_{0}(x) \text { for } 0 \leqslant x \leqslant 1 \\
& f_{\mathrm{b} k}(u)-\left.a(u) \frac{\partial u}{\partial x}\right|_{x=0}=0 \text { for } 0<t \leqslant T \\
& u(1, t)=\bar{u}_{1}(t) \text { for } 0<t \leqslant T .
\end{aligned}
$$

This means that (2.15) is hyperbolic for $u \leqslant \phi_{\mathrm{c}}$, parabolic for $\phi_{\mathrm{c}}<u<1$, and hyperbolic for $u \geqslant 1$. For $u \geqslant 1$, equation (2.15) degenerates into the linear advection equation

$$
\frac{\partial u}{\partial t}+q(t) \frac{\partial u}{\partial x}=0
$$

In Reference 4 it is shown that the generalized solution to the initial-boundary value problem assumes values from the unit interval $[0,1]$ almost everywhere, such that it is sufficient to consider the degeneracy only for $0 \leqslant u \leqslant \phi_{\mathrm{c}}$ and for $u=1$. Apart from that we assume that the smoothness of $\bar{u}_{1}(t)$ implies only a finite number of type changes of (2.15) at $x=1$, i.e. we assume that the time interval $[0, T]$ may be partitioned into a 'hyperbolic' part $H$ and a 'parabolic' part $P$ :

$$
a\left(\bar{u}_{1}(t)\right) \begin{cases}>0 & \text { for } t \in P:=I_{1} \cup \cdots \cup I_{n} \subset[0, T], \\ =0 & \text { for } t \in H:=[0, T] \backslash P,\end{cases}
$$


where $I_{k}$ are intervals or the empty set. Similar boundary value problems for quasilinear parabolic degenerate equations with homogeneous boundary data were studied by $\mathrm{Wu}$ [27] and $\mathrm{Wu}$ and Wang [28]. These results are generalized to the problem under consideration here.

\subsection{Compatibility conditions}

At last, we require that the given initial and boundary data satisfy the following first-order compatibility conditions:

$$
\begin{aligned}
& u_{0}(1)=\bar{u}_{1}(0), \\
& -q(0) u_{0}^{\prime}(1)-f_{\mathrm{b} k}^{\prime}\left(u_{0}(1)\right) u_{0}^{\prime}(1)-\left(a^{\prime}\left(u_{0}(1)\right)\left(u_{0}^{\prime}(1)\right)^{2}\right. \\
& \left.\quad+a\left(u_{0}(1)\right)+u_{0}^{\prime \prime}(1)\right)=\bar{u}_{1}^{\prime}(0), \\
& a\left(u_{0}(0)\right) u_{0}^{\prime}(0)-f_{\mathrm{b} k}\left(u_{0}(0)\right)=0 .
\end{aligned}
$$

These conditions are necessary for the existence of a smooth solution in $C^{2,1}\left(\overline{Q_{T}}\right)$ of the corresponding regularized, parabolic problem. We note that these smoothness and compatibility conditions required will also be assumed for the data of the degenerate problem. The compatibility conditions are used in the existence proof in Reference 4. Here, however, they are only quoted for the purpose of completeness for the statement of the problem. The regularity assumptions might be relaxed, and using a mollifier technique it should be possible to consider discontinuous initial and boundary data. The compatibility conditions can be circumvented, but in this case boundedness of the derivatives of the regularized solution at $(0,0)$ and $(1,0)$ cannot be expected any more.

\subsection{Definition of generalized solutions}

Since a hyperbolic equation might have a discontinuous generalized solution despite smooth initial data, the solutions of the initial-boundary value problem (2.15)-(2.18) must belong to a space of functions allowing discontinuities. Following $\mathrm{Wu}$ [27], we consider generalized, discontinuous solutions in the space $\mathrm{BV}\left(Q_{T}\right)$ of all functions that are defined and summable on $Q_{T}$ and whose generalized first derivatives are Borel measures; and for which

$$
\iint_{\mathrm{Q}_{T}} u \cdot \nabla \varphi \mathrm{d} x \mathrm{~d} t=-\iint_{\mathrm{Q}_{T}} \varphi \cdot\left(\frac{\partial u}{\partial x}(\mathrm{~d} x \mathrm{~d} t), \frac{\partial u}{\partial t}(\mathrm{~d} x \mathrm{~d} t)\right)^{T} \quad \forall \varphi \in C_{0}^{\infty}\left(Q_{T}\right),
$$

where $\nabla \varphi=\left(\varphi_{x}, \varphi_{t}\right)^{T}$.

Theorem 2.1. (Krickeberg and Federer; see [25]). Assume that $u=u(x, t)$ is a bounded function defined and summable on $Q_{T}$. Then $u \in \mathrm{BV}\left(Q_{T}\right)$ if and only if $u$ is of bounded variation in the sense of Tonelli and Cesari, i.e. if the integrals $\int_{0}^{T} \overline{T V}_{x} u(\cdot, t) \mathrm{d} t$ and $\int_{0}^{T} \overline{T V}_{t} u(x, \cdot) \mathrm{d} x$ of the essential total variations of $u$ with respect to each one variable exist. 
The membership of generalized solutions to $\mathrm{BV}\left(Q_{T}\right)$ will be validated by the following useful criterion:

Theorem 2.2. Let $u(x, t)$ be a bounded function defined and summable on $Q_{T}$. Then $u \in \operatorname{BV}\left(Q_{T}\right)$ if and only if there exist constants $K_{1}, K_{2}$ such that the following two inequalities hold:

$$
\begin{aligned}
& \int_{0}^{T} \int_{0}^{1-\Delta x}|u(x+\Delta x, t)-u(x, t)| \mathrm{d} x \mathrm{~d} t \leqslant K_{1} \Delta x \quad \forall x \in[0,1], \\
& \int_{0}^{T-\Delta t} \int_{0}^{1}|u(x, t+\Delta t)-u(x, t)| \mathrm{d} x \mathrm{~d} t \leqslant K_{2} \Delta t \quad \forall \Delta t \in[0, T] .
\end{aligned}
$$

For a detailed account of properties of the function spaces BV, we refer to the work of Vol'pert et al. [23-25]. Denoting by $v=\left(v_{t}, v_{x}\right)$ the normal to $\Gamma_{u}$ and by $u^{ \pm}$the approximate limits of $u$ with respect to $\pm v$, we set for notational convenience $u_{*}:=\min \left\{u^{-}, u^{+}\right\}, u^{*}:=\max \left\{u^{-}, u^{+}\right\}, \bar{u}:=\frac{1}{2}\left(u^{+}+u^{-}\right)$and $\sigma:=\frac{1}{2}\left(\operatorname{sgn} u^{+}+\operatorname{sgn} u^{-}\right)$. Furthermore, let $H=H_{1}$ be the one-dimensional Hausdorff measure. It is possible to derive differentiation formulae for measurable functions in $\mathrm{BV}$ which generalize the common ones for differentiable functions [24]. The product of a measurable function $u$ with a measure $\mu$ is defined as the measure $u \mu$ for which $(u \mu)(E)=\int_{E} u \mathrm{~d} \mu$ for any bounded Borel set $E, E \subset \overline{Q_{T}}$ is valid. However, to keep the chain rule for the differentiation of $(f \circ u)(x)$ valid in $\mathrm{BV}$, the composition has to be replaced by the functional superposition

$$
f\left(\widehat{u(x, t))}:=\int_{0}^{1} f\left(\tau u^{+}(x, t)+(1-\tau) u^{-}(x, t)\right) \mathrm{d} \tau .\right.
$$

The definition of generalized solutions of the initial-boundary value problem is based on an integral inequality with Kružkov entropy functions and corresponding fluxes [17].

Definition 2.1. A function $u \in L^{\infty}\left(Q_{T}\right) \cap B V\left(Q_{T}\right)$ is a generalized solution of the initial-boundary value problem (2.15)-(2.18) if the following conditions are satisfied:

(1) There exists a function $g \in L^{2}\left(Q_{T}\right)$ such that with $r(\tau)=\sqrt{a(\tau)}$ there holds

$$
\iint_{Q_{T}} \varphi g \mathrm{~d} x \mathrm{~d} t=\iint_{Q_{T}} \varphi \widehat{r(u)} \frac{\partial u}{\partial x} \mathrm{~d} x \mathrm{~d} t \quad \forall \varphi \in C_{0}^{\infty}\left(Q_{T}\right) .
$$

(2) The function $u$ satisfies the integral inequality

$$
\begin{array}{r}
\iint_{Q_{T}}\left\{|u-k| \frac{\partial \varphi}{\partial t}+\operatorname{sgn}(u-k)\left[f(u, t)-f(k, t)-\widehat{a(u)} \frac{\partial u}{\partial x}\right] \frac{\partial \varphi}{\partial x}\right\} \mathrm{d} x \mathrm{~d} t \\
+\int_{0}^{T}\left\{-\operatorname{sgn}\left(\bar{u}_{1}(t)-k\right)\left[f(\gamma u, t)-f(k, t)+\gamma\left(\widehat{a(u)} \frac{\partial u}{\partial x}\right)\right] \varphi(1, t)\right. \\
\left.+\left[\operatorname{sgn}(\gamma u-k)-\operatorname{sgn}\left(\bar{u}_{1}(t)-k\right)\right](A(\gamma u)-A(k)) \frac{\partial \varphi}{\partial x}(1, t)\right\} \mathrm{d} t \geqslant 0
\end{array}
$$


for all $\varphi \in C^{\infty}((0,1] \times[0, T])$ where $\varphi \geqslant 0$ and $\operatorname{supp} \varphi \subset(0,1] \times(0, T)$, for all $k \in \mathbb{R}$ and $A(u)=\int_{0}^{u} a(\tau) \mathrm{d} \tau$.

(3) For almost all $t \in[0, T]$ :

(4) For almost all $x \in[0,1]$ :

$$
\left.\gamma\left(-\widehat{a(u)} \frac{\partial u}{\partial x}+f_{\mathrm{b} k}(u)\right)\right|_{x=0}=0 .
$$

$$
(\gamma u)(0, x)=u_{0}(x)
$$

In this definition we use

Lemma 2.1. (Wu and Wang [28]). If $\partial v / \partial t$ and $\partial v / \partial x$ are measures (of bounded variation, which will not be mentioned anymore) for $v(x, t) \in L^{1}\left(Q_{T}\right)$, then for almost all $x \in[0,1]$ and f.a.a. $t \in[0, T]$, the traces $(\gamma v)(x, 0):=\lim _{t \rightarrow 0+} \tilde{v}(x, t),(\gamma v)(0, t):=$ $\lim _{x \rightarrow 0+} \tilde{v}(x, t)$ and $(\gamma v)(1, t):=\lim _{x \rightarrow 1-} \tilde{v}(x, t)$ exist, and $(\gamma v)(x, 0) \in L^{1}(0,1),(\gamma v)(0, t)$, $(\gamma v)(1, t) \in L^{1}(0, T)$, where $\tilde{v}(x, t)$ is a function equivalent to $v(x, t)$ on $Q_{T}$. The traces $\gamma v$ are obviously independent of the chosen function $\tilde{v}$.

These traces are called one one-sided approximate limits.

\section{Jump condition}

The derivation of a jump condition valid on $Q_{T}$ for generalized solutions follows from results for Cauchy problems. The jump condition by Vol'pert and Hudjaev in Reference 24, however, is based on the incorrect assumption that for a function $u \in \operatorname{BV}\left(Q_{T}\right)$ also $\widehat{a}(u) \partial u / \partial x \in \operatorname{BV}\left(Q_{T}\right)$ is valid. It is only true that in this case $(\partial / \partial x)(\widehat{a}(u) \partial u / \partial x)$ is a regular measure of bounded variation on $Q_{T}$. Hence, $\widehat{a}(u) \partial u / \partial x$ belongs to the space $\mathrm{BV}_{x} \supset \mathrm{BV}\left(Q_{T}\right)$ of all functions defined on $Q_{T}$ which for almost all fixed values $t \in(0, T)$ belong to $\mathrm{BV}([0,1])$. From Theorem 2.1 it follows then that $\int_{0}^{T}|\partial u / \partial x(\cdot, t)| \mathrm{d} x$ is integrable on $(0, T)$. The correct jump condition which takes this into account is given as a modification of Vol'pert and Hudjaev's proof by Wu and Yin [29]. It starts from a definition of generalized solutions without initial or boundary conditions:

Definition 3.1. A function $u \in L^{\infty}\left(Q_{T}\right) \cap \mathrm{BV}\left(Q_{T}\right)$ is said to be a generalized solution of equation (2.15) on $Q_{T}$ if the following conditions are satisfied:

(1) There exists a function $g \in L^{1}\left(Q_{T}\right)$ such that

$$
\forall \varphi \in C_{0}^{\infty}\left(Q_{T}\right), \quad \iint_{Q_{T}} \varphi(x, t) g(x, t) \mathrm{d} x \mathrm{~d} t=\iint_{Q_{T}} \varphi(x, t) \widehat{a(u)} \frac{\partial u}{\partial x} \mathrm{~d} x \mathrm{~d} t .
$$

(2) $\forall \varphi \in C_{0}^{\infty}\left(Q_{T}\right), \quad \varphi \geqslant 0, k \in \mathbb{R}$ :

$$
\begin{aligned}
& J(u, k, \varphi):=\frac{1}{2} \iint_{Q_{T}}\left\{|u-k| \frac{\partial \varphi}{\partial t}+\operatorname{sgn}(u-k) \times\right. \\
& \left.\times\left(f(u, t)-f(k, t)-\widehat{a(u)} \frac{\partial u}{\partial x}\right) \frac{\partial \varphi}{\partial x}\right\} \mathrm{d} x \mathrm{~d} t \geqslant 0 .
\end{aligned}
$$


Here condition (3.1) replaces the condition given by Vol'pert and Hudjaev in Reference 24 which we also adopt in our definition of generalized solutions of the initial-boundary value problem. For $r(u):=\sqrt{a(u)}$, their condition reads

$$
\begin{aligned}
& \exists g \in L^{2}\left(Q_{T}\right) \forall \varphi \in C_{0}^{\infty}\left(Q_{T}\right): \\
& \quad \iint_{Q_{T}} \varphi(x, t) g(x, t) \mathrm{d} x \mathrm{~d} t=\iint_{Q_{T}} \varphi(x, t) \widehat{r(u)} \frac{\partial u}{\partial x} \mathrm{~d} x \mathrm{~d} t .
\end{aligned}
$$

Denoting by $u^{\mathrm{r}}(x, t)$ and $u^{\ell}(x, t)$ the right and left approximate limits of $u(\cdot, t)$ as a function of $x$ and setting $\tilde{u}(x, t):=\frac{1}{2}\left(u^{\ell}(x, t)+u^{\mathrm{r}}(x, t)\right)$, the result is

Theorem 3.1. (Wu and Yin [29]). If $u$ is a generalized solution of equation (2.15) on $Q_{T}$, then the following conditions hold $H$-almost everywhere on $\Gamma_{u}$ :

$$
\begin{aligned}
& \left(u^{+}-u^{-}\right) v_{t}+\left(f\left(u^{+}, t\right)-f\left(u^{-}, t\right)\right) v_{x}-\left[\left(\widehat{a(u)} \frac{\partial u}{\partial x}\right)^{\mathrm{r}}-\left(\widehat{a(u)} \frac{\partial u}{\partial x}\right)^{\ell}\right]\left|v_{x}\right|=0 \\
& \forall u \in\left[u_{*}, u^{*}\right]: \quad a(u)=0 \\
& \forall k \in \mathbb{R}: \quad\left[\operatorname{sgn}\left(u^{+}-k\right)-\operatorname{sgn}\left(u^{-}-k\right)\right] \times \\
& \times\left[(\bar{u}-k) v_{t}+(\overline{f(u, t)}-f(k, t)) v_{x}-\left(\widehat{a(u)} \frac{\partial u}{\partial x}\right)^{\sim} v_{x}\right] \leqslant 0
\end{aligned}
$$

As it is shown in Reference 24 that condition (3.1) can be deduced from $u \in L^{\infty}\left(Q_{T}\right)$, (3.5) and (3.3), the generalized solutions of the equation defined in Reference 24 are also generalized solutions in the sense given here in Definition 3.1.

In the formulation of Theorem 3.1, we merely assume $a(u) \geqslant 0$. Using the detailed functional dependency of $a$ on $u$ given by (2.14) and anticipating that the generalized solution of the initial-boundary problem assumes only values between zero and one (see Reference 4), we can formulate the jump condition more instructively:

Corollary 3.1. If $u$ is a generalized solution of the initial-boundary value problem (2.15)-(2.18), then a jump between two values of the solution, $u^{-}$and $u^{+}$, can occur only for $0 \leqslant u^{-}, u^{+} \leqslant \phi_{\mathrm{c}}$. This jump must necessarily satisfy one of the following conditions:

(1) For $0 \leqslant u^{-}, u^{+} \leqslant \phi_{\mathrm{c}}$ the usual Rankine-Hugoniot condition for hyperbolic conservation laws is valid. The propagation velocity s of the jump between $u^{-}$and $u^{+}$ is given by

$$
s=\frac{f\left(u^{+}, t\right)-f\left(u^{-}, t\right)}{u^{+}-u^{-}}=q(t)+\frac{f_{\mathrm{b} k}\left(u^{+}\right)-f_{\mathrm{b} k}\left(u^{-}\right)}{u^{+}-u^{-}} .
$$


Inequality (3.6) then reads

$$
\begin{aligned}
\forall k \in \mathbb{R}: \quad & {\left[\operatorname{sgn}\left(u^{+}-k\right)-\operatorname{sgn}\left(u^{-}-k\right)\right] \times } \\
& \times\left[(\bar{u}-k) v_{t}+(\overline{f(u, t)-f(k, t)}) v_{x}\right] \leqslant 0
\end{aligned}
$$

which is equivalent to Olěnik's jump condition [20],

$$
\forall k \in I\left(u^{-}, u^{+}\right), \quad \frac{f\left(u^{+}, t\right)-f(k, t)}{u^{+}-k} \leqslant s \leqslant \frac{f\left(u^{-}, t\right)-f(k, t)}{u^{-}-k} .
$$

(2) For $0 \leqslant u^{+}<u^{-}=\phi_{\mathrm{c}}$, the velocity of a jump at $\left(x_{0}, t_{0}\right) \in Q_{T}$ is given by

$$
s=\frac{f\left(u^{+}, t_{0}\right)-f\left(\phi_{\mathrm{c}}, t_{0}\right)+\lim _{x \rightarrow x_{0}^{-}} \widehat{a(u) \partial u / \partial x}}{u^{+}-\phi_{\mathrm{c}}}
$$

and inequality (3.6) is equivalent to

$$
\begin{aligned}
& \forall k \in\left[u^{+}, \phi^{\mathrm{c}}\right]: \\
& \frac{f\left(u^{+}, t_{0}\right)-f\left(k, t_{0}\right)}{u^{+}-k} \leqslant s \leqslant \frac{f\left(\phi_{\mathrm{c}}, t_{0}\right)-\lim _{x \rightarrow x_{0}^{-}} \widehat{a(u)(\partial u / \partial x)-f\left(k, t_{0}\right)}}{\phi_{\mathrm{c}}-k} .
\end{aligned}
$$

(3) Analogously, for $0 \leqslant u^{-}<u^{+}=\phi_{\mathrm{c}}$, we obtain

$$
s=\frac{\left.f\left(u^{-}, t_{0}\right)-f\left(\phi_{\mathrm{c}}, t_{0}\right)+\lim _{x \rightarrow x_{0}^{+}} \widehat{a} \widehat{u}\right) \partial u / \partial x}{u^{-}-\phi_{\mathrm{c}}}
$$

and

$$
\begin{aligned}
\forall k & \in\left[u^{-}, \phi_{\mathrm{c}}\right]: \frac{f\left(\phi_{\mathrm{c}}, t_{0}\right)-\lim _{x \rightarrow x_{0}^{+}} \widehat{a}(u) \partial u / \partial x-f\left(k, t_{0}\right)}{\phi_{\mathrm{c}}-k} \leqslant s \\
& \leqslant \frac{f\left(u^{-}, t_{0}\right)-f\left(k, t_{0}\right)}{u^{-}-k} .
\end{aligned}
$$

Proof. Within the proof of existence of a generalized solution of the initial-boundary value problem it is shown in Reference 4 that $0 \leqslant u(x, t) \leqslant 1$ holds on $Q_{T}$, hence the degeneracy of $a(u)$ for $u=1$ does not imply discontinuities of the generalized solution. Relations (3.7), (3.9) and (3.11) immediately follow from $s=-v_{t} / v_{x}$. For the proof of the equivalences of the inequalities, we present only the case (3.10); the other inequalities follow analogously.

“ $\Leftarrow$ ": Multiplying the left inequality of $(3.10)$ by $\operatorname{sgn}\left(u^{+}-k\right) \cdot\left(u^{+}-k\right)$ and the right inequality by $\operatorname{sgn}\left(\phi_{\mathrm{c}}-k\right) \cdot\left(\phi_{\mathrm{c}}-k\right)$, we obtain

$$
\begin{aligned}
& \forall k \in\left[u^{+}, \phi_{\mathrm{c}}\right], \quad \operatorname{sgn}\left(u^{+}-k\right)\left[\left(u^{+}-k\right) v_{t}+\left(f\left(u^{+}, t_{0}\right)-f\left(k, t_{0}\right)\right) v_{x}\right] \leqslant 0 \\
& \wedge \operatorname{sgn}\left(\phi_{\mathrm{c}}-k\right)\left[\left(\phi_{\mathrm{c}}-k\right) v_{t}+\left(f\left(\phi_{\mathrm{c}}, t_{0}\right)-f\left(k, t_{0}\right)-\lim _{x \rightarrow x_{0}^{+}} \widehat{a(u)} \frac{\partial u}{\partial x}\right) v_{x}\right] \leqslant 0 .
\end{aligned}
$$


Using that $\operatorname{sgn}\left(u^{+}-k\right)=-\operatorname{sgn}\left(\phi_{\mathrm{c}}-k\right)$ for $k \in\left[u^{+}, \phi_{\mathrm{c}}\right]$, we have

$$
\begin{aligned}
\forall k \in\left[u^{+}, \phi_{\mathrm{c}}\right]: \quad\left[\operatorname{sgn}\left(u^{+}-k\right)-\operatorname{sgn}\left(\phi_{\mathrm{c}}-k\right)\right] & \times\left[\left(u^{+}-k\right) v_{t}+\left(f\left(u^{+}, t_{0}\right)-f\left(k, t_{0}\right)\right) v_{x}\right] \leqslant 0 \\
\wedge & {\left[\operatorname{sgn}\left(u^{+}-k\right)-\operatorname{sgn}\left(\phi_{\mathrm{c}}-k\right)\right] } \\
\times & {\left[\left(\phi_{\mathrm{c}}-k\right) v_{t}+\left(f\left(\phi_{\mathrm{c}}, t_{0}\right)-f\left(k, t_{0}\right)-\lim _{x \rightarrow x_{0}^{-}} \widehat{a(u)} \frac{\partial u}{\partial x}\right) v_{x}\right] \leqslant 0, }
\end{aligned}
$$

from which (3.6) follows by addition.

“ $\Rightarrow$ ": From (3.6) there follows for $k \in\left[u^{+}, \phi_{\mathrm{c}}\right]$

$$
\begin{aligned}
& \operatorname{sgn}\left(u^{+}-k\right)-\left[\left(u^{+}-k\right) v_{t}-\left(f\left(u^{+}, t_{0}\right)-f\left(k, t_{0}\right)\right) v_{x}\right] \\
& \leqslant \operatorname{sgn}\left(\phi_{\mathrm{c}}-k\right)\left[\left(\phi_{\mathrm{c}}-k\right) v_{t}+\left(f\left(\phi_{\mathrm{c}}, t_{0}\right)-\lim _{x \rightarrow x_{0}^{-}} \widehat{a(u)} \frac{\partial u}{\partial x}-f\left(k, t_{0}\right)\right) v_{x}\right] \\
& \left.=-\operatorname{sgn}\left(u^{+}-k\right)\left[\left(\phi_{\mathrm{c}}-u^{+}\right) v_{t}+\left(f\left(\phi_{\mathrm{c}}, t_{0}\right)-\lim _{x \rightarrow x_{0}^{-}} \widehat{a(u}\right) \frac{\partial u}{\partial x}-f\left(u^{+}, t_{0}\right)\right) v_{x}\right] \\
& \quad-\operatorname{sgn}\left(u^{+}-k\right)\left[\left(u^{+}-k\right) v_{t}+\left(f\left(u^{+}, t_{0}\right)-f\left(k, t_{0}\right)\right) v_{x}\right] .
\end{aligned}
$$

From (3.9) we obtain

$$
\left.\left(\phi_{\mathrm{c}}-u^{+}\right) v_{t}+\left(f\left(\phi_{\mathrm{c}}, t_{0}\right)-\lim _{x \rightarrow x_{0}^{-}} \widehat{a(u}\right) \frac{\partial u}{\partial x}-f\left(u^{+}, t_{0}\right)\right) v_{x}=0
$$

and hence

$$
\operatorname{sgn}\left(u^{+}-k\right)\left[\left(u^{+}-k\right) v_{t}+\left(f\left(u^{+}, t_{0}\right)-f\left(k, t_{0}\right)\right) v_{x}\right] \leqslant 0,
$$

from which the left inequality of (3.10) follows by division by $\operatorname{sgn}\left(u^{+}-k\right)\left(u^{+}-k\right)$. The right inequality follows by the same arguments.

\section{Entropy boundary condition}

\subsection{Preliminary remark}

From (2.25) we can infer that the measure $\widehat{r(u)} \partial u / \partial x$ (and hence the measure $\widehat{a(u)} \partial u / \partial x)$ is absolutely continuous, such that, by the Radon-Nikodym theorem, there exists a function $h \in L^{1}\left(Q_{T}\right)$ such that

$$
\left(\widehat{a(u)} \frac{\partial u}{\partial x}\right)(E)=\int_{E} h \mathrm{~d} x \mathrm{~d} t \quad \text { for all Borel sets } E .
$$

The notation

$$
\iint_{Q_{T}} \varphi h \mathrm{~d} x \mathrm{~d} t=\iint_{Q_{T}} \varphi \widehat{a(u)} \frac{\partial u}{\partial x} \mathrm{~d} x \mathrm{~d} t \quad \forall \varphi \in C_{0}^{\infty}\left(Q_{T}\right)
$$


justifies identifying $\widehat{a(u)} \partial u / \partial x$ with $h$. Furthermore, we need the existence of the trace $\gamma(\widehat{a(u)} \partial u / \partial x)$ in $(2.26)$, for which it is sufficient to require that $(\partial u / \partial x)(\widehat{a}(u) \partial u / \partial x)$ is a measure.

\subsection{The entropy boundary inequality}

To obtain a well-posed problem, we now derive an entropy boundary condition for $x=1$ extending the results of References 1,10 and 16 where the case of a hyperbolic equation was investigated. This entropy boundary condition leads to a set-valued reformulation of the boundary condition at $x=1$, by which we take into account that for large times the boundary datum $\bar{u}_{1}(t)$ stipulated at $x=1$ need not be assumed exactly by the generalized solution.

This phenomenon can be illustrated easily if we recall the 'fan structure' of the entropy solution of the Riemann problem of a hyperbolic equation on the spatially bounded domain $Q_{T}$, where we assume the initial jump to be situated at, say, $x=0 \cdot 5$. In this case, the Riemann data will be assumed by the entropy solution at $x=0$ and 1 , respectively, only for a finite time interval.

In the following, we derive an inequality which is valid at $x=1$ in any case relating the value the generalized solution actually assumes, $(\gamma u)(1, t)$, to the boundary datum $\bar{u}_{1}(t)$, which is prescribed at $(1, t)$ by the boundary condition (2.18). The pertinent set-valued reformulation of boundary condition (2.18), its geometrical visualization and its characterization as a set of possible solution values are given subsequently.

Now, we choose in (2.26) special test functions. Let $\delta \in C^{\infty}(\mathbb{R})$ with

$$
\delta(\sigma) \geqslant 0, \quad \delta(\sigma)=0 \quad \text { for }|\sigma| \geqslant 1, \quad \int_{-\infty}^{\infty} \delta(\sigma) \mathrm{d} \sigma=1
$$

and set for $h>0$

$$
\begin{aligned}
& \delta_{h}(\sigma):=\frac{1}{h} \delta\left(\frac{\sigma}{h}\right), \quad \varrho_{h}(\sigma):=\int_{-\infty}^{\sigma} \delta_{h}(\tau) \mathrm{d} \tau, \\
& \mu_{h}(\sigma):=1-\varrho_{h}(\sigma-2 h), \quad v_{h}(\sigma):=\varrho_{h}(\sigma-(1-2 h)) .
\end{aligned}
$$

For these test functions, the following lemmata are valid given in Reference 28:

Lemma 4.1. Let $v(x, t) \in L^{1}\left(Q_{T}\right)$ and $|v(x, t)| \leqslant \Phi(t)$ a.e. in $Q_{T}$ with a function $\Phi(t) \in L^{1}(0, T)$. If $(\gamma v)(0, t)$ and $(\gamma v)(1, t)$ exist a.e. in $[0, T]$, then we have for $\varphi \in C^{\infty}\left(Q_{T}\right)$ :

$$
\begin{aligned}
& \lim _{h \rightarrow 0^{+}} \iint_{Q_{T}} \frac{\partial}{\partial x}\left(\varphi(x, t) \mu_{h}(x)\right) v(x, t) \mathrm{d} x \mathrm{~d} t=-\int_{0}^{T} \varphi(0, t)(\gamma v)(0, t) \mathrm{d} t, \\
& \lim _{h \rightarrow 0^{+}} \iint_{Q_{T}} \frac{\partial}{\partial x}\left(\varphi(x, t) v_{h}(x)\right) v(x, t) \mathrm{d} x \mathrm{~d} t=\int_{0}^{T} \varphi(1, t)(\gamma v)(1, t) \mathrm{d} t .
\end{aligned}
$$


Lemma 4.2. If $v(x, t) \in L^{1}\left(Q_{T}\right)$ and $\partial v / \partial x$ is an absolutely continuous measure, then for $\varphi \in C^{\infty}\left(\overline{Q_{T}}\right)$ with $\varphi \subset[0,1] \times(0, T)$ there holds

$$
\iint_{Q_{T}} \frac{\partial v}{\partial x} v \mathrm{~d} t \mathrm{~d} x=\left.\int_{0}^{T} \varphi \cdot \gamma v\right|_{x=0} ^{x=1} \mathrm{~d} t-\iint_{Q_{T}} \varphi \frac{\partial v}{\partial x} \mathrm{~d} t \mathrm{~d} x .
$$

Lemma 4.3. If $v(x, t) \in L^{1}\left(Q_{T}\right)$ and $\partial v / \partial x$ is a measure, then the trace $\gamma \operatorname{sgn}(v-k)$ exists for almost all $k \in \mathbb{R}$ almost everywhere on $[0, T]$, and

$$
\left.\gamma \operatorname{sgn}(v-k)\right|_{x=0,1}=\left.\operatorname{sgn}(\gamma v-k)\right|_{x=0,1} .
$$

Now, if we choose in the integral inequality (2.26) as test function $\varphi(x) v_{h}(x) \psi(t)$ with $\varphi(x) \geqslant 0, \varphi \in C_{0}^{\infty}[0,1], \psi(t) \geqslant 0, \psi \in C_{0}^{\infty}[0, T]$ and $v_{h}(x)$ from (4.2), then by

$$
\iint_{Q_{T}}|u-k| \frac{\partial}{\partial t}\left(\varphi(x) v_{h}(x) \psi(t)\right) \mathrm{d} x \mathrm{~d} t \stackrel{h \rightarrow 0}{\rightarrow} 0
$$

and (application of Lemmas 4.1 and 4.3)

$$
\begin{aligned}
& \iint_{Q_{T}} \operatorname{sgn}(u-k)\left[f(u, t)-f(k, t)-\widehat{a(u)} \frac{\partial u}{\partial x}\right] \psi(t) \varphi(x) v_{h}^{\prime}(x) \mathrm{d} x \mathrm{~d} t \\
& \left.\stackrel{h \rightarrow 0}{\rightarrow} \int_{0}^{T} \operatorname{sgn}(\gamma u-k)\left[f(\gamma u, t)-f(k, t)-\gamma\left(\widehat{a(u)} \frac{\partial u}{\partial x}\right)\right] \psi(t) \varphi(x)\right|_{x=1} \mathrm{~d} t,
\end{aligned}
$$

we can derive from the entropy inequality (2.26) the inequality

$$
\begin{aligned}
\int_{0}^{T}\{( & \left.\operatorname{sgn}(\gamma u-k)-\operatorname{sgn}\left(\bar{u}_{1}(t)-k\right)\right)(A(\gamma u)-A(k)) \psi(t) \varphi^{\prime}(x) \\
& +\left[\operatorname{sgn}(\gamma u-k)-\operatorname{sgn}\left(\bar{u}_{1}(t)-k\right)\right] \\
\quad & {\left.\left.\left[f(\gamma u, t)-f(k, t)-\gamma\left(\widehat{a(u)} \frac{\partial u}{\partial x}\right)\right] \cdot \psi(t) \varphi(x)\right|_{x=1}\right\} \mathrm{d} t \geqslant 0 . }
\end{aligned}
$$

Due to the arbitrariness of the test function $\psi(t)$, the following inequality holds for almost all $k \in \mathbb{R}$ almost everywhere on $[0, T]$ :

$$
\begin{aligned}
& {\left.\left[\operatorname{sgn}(\gamma u-k)-\operatorname{sgn}\left(\bar{u}_{1}(t)-k\right)\right][A(\gamma u)-A(k)] \varphi^{\prime}\right|_{x=1}} \\
& \quad+\left[\operatorname{sgn}(\gamma u-k)-\operatorname{sgn}\left(\bar{u}_{1}(t)-k\right)\right] \\
& \quad \times\left.\left[f(\gamma u, t)-f(k, t)-\gamma\left(\widehat{a}(u) \frac{\partial u}{\partial x}\right)\right] \varphi\right|_{x=1} \geqslant 0 .
\end{aligned}
$$

As $\varphi$ was chosen arbitrarily as well, $\varphi^{\prime}$ can assume arbitrary values. Hence, we can conclude for the first term in (4.7) defining $I(a, b):=[\min \{a, b\}, \max \{a, b\}]$ :

$$
\begin{aligned}
& {\left.\left[\operatorname{sgn}(\gamma u-k)-\operatorname{sgn}\left(\bar{u}_{1}(t)-k\right)\right][A(\gamma u)-A(k)]\right|_{x=1}=0,} \\
& \text { f.a.a. } k \in I\left(\bar{u}_{1}(t),(\gamma u)(1, t)\right) \quad \text { a.e. in }[0, T]
\end{aligned}
$$

which can be written as

$$
a(\tau)=0 \quad \text { for all } \tau \in I\left(\bar{u}_{1}(t),(\gamma u)(1, t)\right) .
$$


From the separate entropy boundary condition valid at $x=1$, combined with the Kružkov integral inequality for test functions that vanish on all boundaries of $Q_{T}$, integral inequality (2.26) can be derived. Summing up, we arrive at

Lemma 4.4. Condition (2.26) in the definition of the generalized solution is satisfied if and only if integral equality (3.2), condition (4.9) and the following entropy boundary inequality are satisfied: For almost all $k \in \mathbb{R}$ there holds almost everywhere on $[0, T]$

$$
\left.\left[\operatorname{sgn}(\gamma u-k)-\operatorname{sgn}\left(\bar{u}_{1}(t)-k\right)\right]\left[f(\gamma u, t)-f(k, t)-\gamma\left(\widehat{a(u)} \frac{\partial u}{\partial x}\right)\right]\right|_{x=1} \geqslant 0 .
$$

Consequently, the boundary datum at $x=1$, viz., the function $\bar{u}_{1}(t)$, is always assumed exactly for $1>\bar{u}_{1}(t)>\phi_{\mathrm{c}}$, i.e. for $a\left(\bar{u}_{1}(t)\right)>0$. This is also valid for $\bar{u}_{1}(t)=1$, if we anticipate that the generalized solution only assumes values $u \in[0,1]$, which is shown in Reference 4. Otherwise, relation (4.10) is valid.

\subsection{Reformulation of the boundary condition at $x=1$}

Lemma 4.5. (Wu and Wang [28]). Let $0<\alpha \leqslant 1$ be a constant and $K_{\alpha}$ be the subclass of $\operatorname{BV}\left(Q_{T}\right)$ with the additional properties that every $u \in K_{\alpha}$ is bounded and the measure $\mu_{\alpha}=a^{1-\alpha}(u)$ is absolutely continuous at all points where $u$ is approximately continuous. Now, let $a^{\alpha}(u)$ be locally Lipschitz continuous and $u \in K_{\alpha}$ be a generalized solution of (2.15)-(2.18). Then, for almost all $t \in H$,

$$
\gamma\left(\widehat{a(u)} \frac{\partial u}{\partial x}\right)(1, t)=0 .
$$

Under the assumptions of section 2, we have $a(u) \in C^{1}[0,1]$, which means, in particular, that $a(u)$ is Lipschitz continuous, and that $\alpha=1$ can be chosen. Thus, the

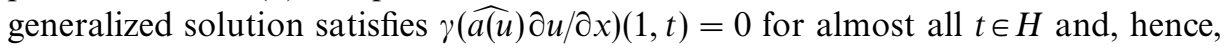

$$
\begin{aligned}
& {\left.\left[\operatorname{sgn}(\gamma u-k)-\operatorname{sgn}\left(\bar{u}_{1}(t)-k\right)\right][f(\gamma u, t)-f(k, t)]\right|_{x=1} \geqslant 0} \\
& \quad \text { f.a.a. } k \in \mathbb{R} \quad \text { a.e. on }[0, T] \\
& \left.\Leftrightarrow \operatorname{sgn}\left((\gamma u)(1, t)-\bar{u}_{1}(t)\right)[f(\gamma u, t)-f(k, t)]\right|_{x=1} \geqslant 0 \\
& \forall k \in I\left((\gamma u)(1, t), \bar{u}_{1}(t)\right) \\
& \Leftrightarrow \frac{f((\gamma u)(1, t), t)-f(k, t)}{(\gamma u)(1, t)-k} \geqslant 0 \quad \forall k \in I\left((\gamma u)(1, t), \bar{u}_{1}(t)\right) .
\end{aligned}
$$

This means that for $t \in H$ the same entropy boundary condition holds as for the hyperbolic initial-boundary value problem that was studied in Reference 10, and that for $t \in H$ the pointwise condition $(\gamma u)(1, t)=\bar{u}_{1}(t)$ does not hold in general. Consequently, the boundary condition at $x=1$ must be reformulated in a set-valued manner, which means that to every value $\bar{u}_{1}(t)$ with $t \in H$, the set of admissible 
boundary values

$$
\mathscr{E}(t):=\left\{u \in[0,1] \mid \frac{f(u, t)-f(k, t)}{u-k} \geqslant 0 \quad \forall k \in I\left(u, \bar{u}_{1}(t)\right)\right\}
$$

is assigned. Hence, the initial-boundary value problem is not properly posed unless we replace condition $(2.18)$ by

$$
u(1, t)\left\{\begin{array}{lll}
=\bar{u}_{1}(t) & \text { if } & \bar{u}_{1}(t)>\phi_{\mathrm{c}} \\
\in \mathscr{E}(t) & \text { if } & \bar{u}_{1}(t) \leqslant \phi_{\mathrm{c}}, \mathscr{E}(t) \text { from }(4.12) .
\end{array}\right.
$$

The set $\mathscr{E}(t)$ can be visualized for a fixed time $t$ using the graph of $f(u, t)$, see Figures 2 and 3.

Moreover, the set $\mathscr{E}(t)$ can be characterized as the set of possible values of the entropy solution of a Riemann problem of a hyperbolic conservation law:

Theorem 4.1. Let $R_{T}:=\mathbb{R} \times(0, T), v \in L^{\infty}\left(R_{T}\right) \cap \mathrm{BV}\left(R_{T}\right)$ be the entropy solution of the hyperbolic initial value problem

$$
\begin{aligned}
& \frac{\partial}{\partial t} v(x, t)+\frac{\partial}{\partial x} g(v)=0, \quad x \in \mathbb{R}, t \in(0, T), \\
& v(0, t)=v_{0}(x)=\left\{\begin{array}{rll}
v_{0}^{-}=(\gamma u)\left(1, t_{0}\right) & \text { for } & x<0 \\
v_{0}^{+}=\bar{u}_{1}\left(t_{0}\right) & \text { for } & x>0
\end{array}\right.
\end{aligned}
$$

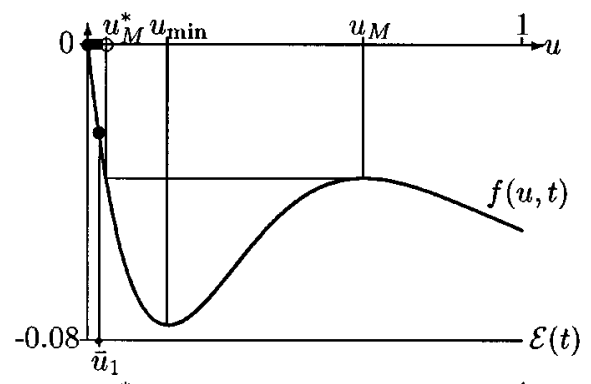

If $0<u_{M} \leq 1$ is the local maximum of $f(u, t)$ and $0 \leq u_{M}^{*}<u_{M}$ with $f\left(u_{M}^{*}, t\right)=f\left(u_{M}, t\right)$ then

$$
\begin{gathered}
\bar{u}_{1}(t) \in\left[0, u_{M}^{*}\right) \Longrightarrow \\
\mathcal{E}(t)=\left\{\bar{u}_{1}(t)\right\} .
\end{gathered}
$$

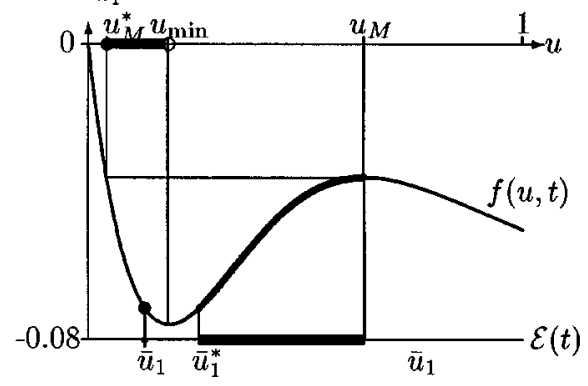

If $0<u_{\min } \leq 1$ is the local minimum of $f(u, t)$ and $f\left(\bar{u}_{1}(t), t\right)=$ $f\left(\bar{u}_{1}^{*}, t\right)$ with $u_{\min }<\bar{u}_{1}^{*}<u_{M}$, then

$$
\begin{gathered}
\bar{u}_{1}(t) \in\left[u_{M}^{*}, u_{\min }\right) \Longrightarrow \\
\mathcal{E}(t)=\left[\bar{u}_{1}^{*}, u_{M}\right] \cup\left\{\bar{u}_{1}(t)\right\} .
\end{gathered}
$$

Fig. 2. The set $\mathscr{E}(t)$ of admissible boundary values. Example: $f(u, t)=-0 \cdot 05 u-u(1-u)^{2}$ 


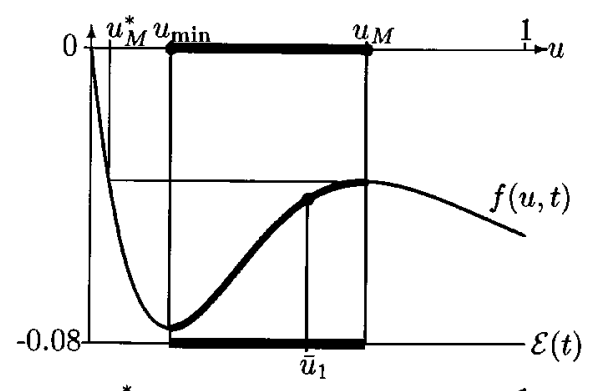

$$
\bar{u}_{1}(t) \in\left[u_{\min }, u_{M}\right] \Longrightarrow
$$$$
\mathcal{E}(t)=\left[u_{\min }, u_{M}\right] \cup\left\{\bar{u}_{1}(t)\right\}
$$

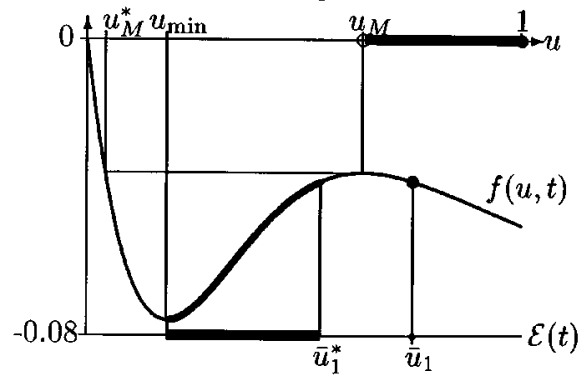

If $u_{M}<\bar{u}_{1}(t) \leq 1$ and $f\left(\bar{u}_{1}(t), t\right)=f\left(u_{1}^{*}, t\right)$ with $u_{\min }<$ $\bar{u}_{1}^{*}<u_{M}$, then

$$
\begin{gathered}
\bar{u}_{1}(t) \in\left(u_{M}, 1\right] \Longrightarrow \\
\mathcal{E}(t)=\left[u_{\min }, \bar{u}_{1}^{*}\right] \cup\left\{\bar{u}_{1}(t)\right\}
\end{gathered}
$$

Fig. 3. The set $\mathscr{E}(t)$ of admissible boundary values (continued)

(Riemann problem) using $g(v):=f\left(v ; t_{0}\right)$. Then there holds

$$
\left\{(\gamma v)\left(0^{-}, t\right) \mid t>0 \wedge v_{0}^{-} \in[0,1]\right\}=\mathscr{E}\left(t_{0}\right) .
$$

Proof. The entropy solution of problem (4.14), (4.15) is defined by [17]

(1) For all $\varphi \in C_{0}^{\infty}\left(R_{T}\right), \varphi \geqslant 0$ there holds for all $k \in \mathbb{R}$

$$
\iint_{R_{T}}\left\{|v-k| \frac{\partial \varphi}{\partial x}+\operatorname{sgn}(v-k)(g(v)-g(k)) \frac{\partial \varphi}{\partial x}\right\} \mathrm{d} x \mathrm{~d} t \geqslant 0 .
$$

(2) For almost all $x \in \mathbb{R},(\gamma v)(x, 0)=v_{0}(x)$.

Now, for a constant $M>0$ set

$$
\lambda_{h}(x ; M):=\left\{\begin{array}{lll}
\mu_{h}(-x) & \text { for } \quad x<0, \\
\mu_{h}(x-M) & \text { for } \quad x \geqslant 0,
\end{array}\right.
$$

using $\mu_{h}$ from (4.2) and $\varphi(x, t):=\varphi(x) \psi(t) \lambda_{h}(x ; M)$ with $\varphi \in C_{0}^{\infty}(\mathbb{R}), \varphi(x) \geqslant 0$, $\psi \in C_{0}^{\infty}(0, T)$, and $\psi \geqslant 0$. From integral inequality (4.17) it follows for $h \rightarrow 0$ that for all $M>0$ there holds for all $k \in \mathbb{R}$ :

$$
\begin{aligned}
& \int_{0}^{T}\left\{-\operatorname{sgn}\left((\gamma v)\left(M^{+}, t\right)-k\right)\left(g(\gamma u)\left(M^{+}, t\right)-g(k)\right) \varphi(M)\right. \\
& \left.\quad+\operatorname{sgn}\left((\gamma v)\left(0^{-}, t\right)-k\right)\left(g(\gamma v)\left(0^{-}, t\right)-g(k)\right) \varphi(0)\right\} \psi(t) \mathrm{d} t \geqslant 0
\end{aligned}
$$


and, hence, for almost all $t \in(0, T)$

$$
\begin{aligned}
& {\left[-\operatorname{sgn}\left((\gamma v)\left(M^{+}, t\right)-k\right)\left(g\left((\gamma v)\left(M^{+}, t\right)\right)-g(k)\right)\right.} \\
& \left.\quad+\operatorname{sgn}\left((\gamma v)\left(0^{-}, t\right)-k\right)\left(g\left((\gamma v)\left(0^{-}, t\right)\right)-g(k)\right)\right] \varphi(0) \\
& \left.\geqslant \operatorname{sgn}\left((\gamma v)\left(M^{+}, t\right)-k\right)\left(g(\gamma v)\left(M^{+}, t\right)\right)-g(k)\right)(\varphi(M)-\varphi(0)) \quad \forall k \in \mathbb{R} .
\end{aligned}
$$

Then for $t>0$ we choose $M=c \cdot t$ with $c>\max _{0 \leqslant u \leqslant 1}\left|f_{u}\left(u, t_{0}\right)\right|$, such that $(M, t)$ is not contained in the 'Riemann fan' representing the similarity solution of problem (4.14), (4.15) (see e.g. Reference 19), and $(\gamma v)\left(M^{+}, t\right)=v_{0}^{+}=\bar{u}_{1}\left(t_{0}\right)$ is valid. Then

$$
\begin{aligned}
& {\left[-\operatorname{sgn}\left(\bar{u}_{1}\left(t_{0}\right)-k\right)\left(g\left(\bar{u}_{1}\left(t_{0}\right)\right)-g(k)\right)\right.} \\
& \left.\quad+\operatorname{sgn}\left((\gamma v)\left(0^{-}, t\right)-k\right)\left(g\left((\gamma v)\left(0^{-}, t\right)\right)-g(k)\right)\right] \varphi(0) \\
& \geqslant \operatorname{sgn}\left(\bar{u}_{1}\left(t_{0}\right)-k\right)\left(g\left(\bar{u}_{1}\left(t_{0}\right)\right)-g(k)\right)(\varphi(c t)-\varphi(0)) \quad \forall k \in \mathbb{R}
\end{aligned}
$$

The right-hand side vanishes for $t \rightarrow 0$ and we obtain from $\varphi \geqslant 0$ that for $\tau>0$ (for $t>0,(\gamma v)\left(0^{-}, t\right)$ is independent of $\left.t\right)$

$$
\begin{aligned}
& -\operatorname{sgn}\left(\bar{u}_{1}\left(t_{0}\right)-k\right)\left(g\left(\bar{u}_{1}\left(t_{0}\right)\right)-g(k)\right) \\
& +\operatorname{sgn}\left((\gamma v)\left(0^{-}, \tau\right)-k\right)\left(g\left((\gamma v)\left(0^{-}, \tau\right)\right)-g(k)\right) \geqslant 0 \quad \forall k \in \mathbb{R}
\end{aligned}
$$

is valid. Here it is sufficient to require that (4.18) holds for all $k \in I\left(\bar{u}_{1}\left(t_{0}\right),(\gamma v)\left(0^{-}, t\right)\right)$. But for these values of $k$, we have

$$
\begin{aligned}
- & \operatorname{sgn}\left(\bar{u}_{1}\left(t_{0}\right)-k\right)\left(g\left(\bar{u}_{1}\left(t_{0}\right)\right)-g(k)\right) \geqslant 0 \\
& \left.\Leftrightarrow \operatorname{sgn}\left((\gamma v)\left(0^{-}, \tau\right)-k\right)\left(g(\gamma v)\left(0^{-}, t\right)\right)-g(k)\right) \geqslant 0 \\
\Leftrightarrow & \frac{f\left((\gamma v)\left(0^{-}, \tau\right), t_{0}\right)-f\left(k, t_{0}\right)}{(\gamma v)\left(0^{-}, \tau\right)-k} \geqslant 0
\end{aligned}
$$

and hence the desired result,

$$
(\gamma v)\left(0^{-}, t\right) \in\left\{u \in[0,1] \mid \frac{f\left(u, t_{0}\right)-f\left(k, t_{0}\right)}{u-k} \geqslant 0 \quad \forall k \in I\left(u, \bar{u}_{1}\left(t_{0}\right)\right)\right\}=\mathscr{E}\left(t_{0}\right) .
$$

\section{Acknowledgements}

This work was made possible by the 'Graduiertenkolleg GKKS' at the University of Stuttgart and by the Collaborative Research Programme (Sonderforschungsbereich) 404 (project TP A2) of the German Research Foundation (DFG).

\section{References}

1. Bardos, C., Le Roux, A. Y. and Nedelec, J. C., 'First order quasilinear equations with boundary conditions', Commun. Partial Differential Equations, 4(9), 1017-1034 (1979).

2. Been, K. and Sills, G. C., 'Self-weight consolidation of soft soils: an experimental and theoretical study', Geotechnique, 31, 519-535 (1981). 
3. Bürger, R., 'Ein Anfangs-Randwertproblem einer quasilinearen entarteten parabolischen Gleichung in der Theorie der Sedimentation mit Kompression', Doctoral Dissertation, University of Stuttgart, Germany, 1996.

4. Bürger, R. and Wendland, W. L., 'Existence, stability and uniqueness of generalized solutions of an initial-boundary value problem for a degenerating quasilinear parabolic equation', preprint 97-39, SFB 404, University of Stuttgart, 1997; J. Math. Anal. Appl., to appear.

5. Bustos, M. C. and Concha, F., 'Simulation of batch sedimentation with compression', A.I.Ch.E. J., 34(5), 859-861 (1988).

6. Bustos, M. C. and Concha, F., 'Kynch theory of sedimentation; in: Sedimentation of Small Particles in a Viscous Fluid, (E. Tory, Ed.), Computational Mechanics Publications, Southampton, U.K., 1996, pp. $7-49$.

7. Bustos, M. C., Concha, F. and Wendland, W. L., 'Global weak solutions to the problem of continuous sedimentation of an ideal suspension', Math. Meth. in the Appl. Sci., 13, 1-22 (1990).

8. Bustos, M. C., Concha, F. and Wendland, W. L., 'Sedimentation with compression. Part II: numerical solution', University of Concepción, 1992.

9. Bustos, M. C., Paiva, F. and Wendland, W. L., 'Control of continuous sedimentation of ideal suspensions as an initial and boundary value problem', Math. Meth. in the Appl. Sci., 12, 533-548 (1990).

10. Bustos, M. C., Paiva, F. and Wendland, W. L., 'Entropy boundary conditions in the theory of sedimentation of ideal suspensions', Math. Meth. in the Appl. Sci., 19, 679-697 (1996).

11. Cheuquepán, F., Paiva, F. and Bustos, M.C., 'Una condición de Rankine-Hugoniot para el problema de sedimentación con compresión', Manuscript, University of Concepción, Chile, 1990.

12. Concha, F. and Bustos, M. C., 'Settling velocities of particulate systems: 6. Kynch sedimentation processes: Batch settling', Int. J. Min. Proc., 32, 193-212 (1991).

13. Concha, F. and Bustos, M. C., 'Settling velocities of particulate systems: 7. Kynch sedimentation processes: continuous thickening', Int. J. Min. Proc., 34, 33-51 (1992).

14. Concha, F., Bustos, M. C. and Barrientos, A., 'Phenomenological theory of sedimentation', in: Sedimentation of Small Particles in a Viscous Fluid, (E. Tory, ed.), Computational Mechanics Publications, Southampton, U.K., 1996, pp. 51-96.

15. Concha, F., Bustos, M. C., Oelker, E. and Wendland, W. L., 'Settling velocities of particulate systems: 9. Phenomenological theory of sedimentation processes I: Batch sedimentation', Int. J. Min. Proc., submitted.

16. Dubois, F. and Le Floch, P., 'Boundary conditions for nonlinear hyperbolic systems of conservation laws', J. Differential Equations, 71, 93-122 (1988).

17. Kružkov, S. N., 'First order quasilinear equations in several independent variables', Math. USSR Sbornik, 10(2), 217-243 (1970).

18. Kynch, G. J., 'A theory of sedimentation', Trans. Farad. Soc., 48, 166-176 (1952).

19. Le Veque, R. J., Numerical Methods for Conservation Laws, 2nd edn, Birkhäuser Verlag, Basel, 1992.

20. Oleinik, O. A., 'Uniqueness and stability of the generalized solution of the Cauchy problem for a quasi-linear equation', AMS Trans. Ser. 2, 33, 285-290 (1963).

21. Richardson, J. F. and Zaki, W. N., 'The sedimentation of uniform spheres under conditions of viscous flow', Chem. Engrg. Sci., 3, 65-73 (1954).

22. Schiffman, R. L., Pane, V. and Gibson, R. E., 'The theory of one-dimensional consolidation of saturated clays, IV. An overview of nonlinear finite strain sedimentation and consolidation', in: Sedimentation and Consolidation models, Predictions and Validations, Proc. ASCE Symp. (R. Yong, ed.), San Francisco; New York 1984.

23. Vol'pert, A. I., 'The spaces BV and quasilinear equations', Math. USSR Sbornik, 2(2), 225-267 (1967).

24. Vol'pert, A. I. and Hudjaev, S. I., 'Cauchy's problem for degenerate second order quasilinear parabolic equations', Math. USSR Sbornik, 7(3), 365-387 (1969).

25. Vol'pert, A. I. and Hudjaev, S. I., Analysis in Classes of Discontinuous Functions and Equations of Mathematical Physics, Martinus Nijhoff Publishers, Dordrecht 1985.

26. $\mathrm{Wu}$, Zhuoqun, 'A note on the first boundary value problem for quasilinear degenerate parabolic equations', Acta Math. Sci., 4(2), 361-373 (1982).

27. $\mathrm{Wu}, \mathrm{Z}$., 'A boundary value problem for quasilinear degenerate parabolic equations', University of Wisconsin, MRC Technical Summary Report \# 2484, 1983.

28. Wu, Z. and Wang, J.-Y, 'Some results on quasilinear degenerate parabolic equations of second order', Proc. 1980 Beijing Symp. on Differential Geometry and Differential Equations, Vol. 3, Science Press, Beijing, Gordon \& Breach, Science Publishers Inc., New York, 1982.

29. Wu, Z. and Yin, J., 'Some properties of functions in $\mathrm{BV}_{x}$ and their applications to the uniqueness of solutions for degenerate quasilinear parabolic equations', Northeastern Math. J., 5(4), 395-422 (1989). 